\title{
Caracterização das condições de saúde e do risco de quedas em uma população idosa
}

\author{
Rafela Simon Myra*, Mariângela De Marco**, Caroline Zanin ${ }^{\star \star}$, Vinícius Dal Molin ${ }^{\star \star *}$, Lia Mara Wibelinger***
}

\section{Resumo}

As alterações decorrentes do processo de envelhecimento, evidenciadas pela diminuição da força muscular, da amplitude de movimento, da velocidade de contração muscular, da acuidade visual e auditiva e pelas alterações posturais influenciam a mobilidade funcional e o equilíbrio em idosos. Essas alterações podem acarretar alguns prejuízos para o idoso, como o aumento do risco de quedas e a redução do nível de independência funcional. A amostra foi composta por 76 idosos, a maioria do gênero feminino, na faixa etária de 60 a 69 anos, casada, com baixas escolaridade e renda. Os indivíduos portam alguma doença associada, sendo a hipertensão arterial sistêmica a mais relatada, fazem uso de medicamentos e de óculos para correção de problemas visuais. A maior parte dos idosos que sofreu quedas nos últimos seis meses apresentava um ou mais fatores de risco para quedas, evidenciando a probabilidade de que o uso de medicamento, a presença de fatores de risco cardiovascular e alterações visuais podem aumentar, efetivamente, o risco de quedas nessa população.

Palavras-chave: Acidente por quedas. Idoso. Qualidade de vida.

\section{Introdução}

A Organização Mundial da Saúde define pessoa idosa como o indivíduo com 60 anos ou mais de idade. Nos últimos anos, a população idosa está crescendo devido às melhorias nas condições de vida e aos avanços da ciência, o que, consequentemente, aumenta a expectativa de vida (SANTOS; BARROS, 2008). No Brasil, projeções do Instituto Brasileiro de Geografia e Estatística indicam que até o ano de 2025, a população idosa, que atualmente inclui $13 \%$ da população total corresponderá a mais de 32 milhões de pessoas (IBGE, 2010).

As alterações decorrentes do processo de envelhecimento, evidenciadas pela diminuição da força muscular, da amplitude de movimento, da velocidade de contração muscular, da acuidade visual e auditiva e pelas alterações posturais influenciam a mobilidade funcional e o

* Acadêmica de Fisioterapia na Universidade de Passo Fundo. Passo Fundo, RS, Brasil. Bolsista Pibic/CNPq. Endereço para correspondência: Rua Coroados, 637, Carazinho, Rio Grande do Sul, CEP 99500-000. E-mail: rafaelasimonmyra@gmail.com

** Acadêmicas de Fisioterapia, Universidade de Passo Fundo. E-mail: mariangelademarco@gmail.com; caroline. zanin@hotmail.com

**** Acadêmico de Fisioterapia. Universidade de Passo Fundo. Bolsista Probic/Fapergs. E-mail: vini.fisio@gmail.com

***** Doutora em Gerontologia Biomédica pela Pontifícia Universidade Católica do Rio Grande do Sul. Docente do curso de Fisioterapia e do Programa de Pós-Graduação em Envelhecimento Humano, Universidade de Passo Fundo. E-mail: liafisio@upf.br

$\rightarrow$ http://dx.doi.org/10.5335/rbceh.v13i1.4868

Recebido em: 19/03/2015. Aceito em: 20/07/2016. 
equilíbrio em idosos. Essas alterações podem acarretar alguns prejuízos para o idoso, como o aumento do risco de quedas e redução do nível de independência funcional (PINHO et al., 2012).

O aumento da população idosa também está relacionado ao crescimento das doenças crônico-degenerativas o que provoca uma série de desafios para a sociedade, além da preparação e adequação dos serviços de saúde, incluindo a formação e capacitação de profissionais para o atendimento dessa nova demanda (PRESTA et al., 2011).

Atualmente, as quedas de idosos constituem uma das preocupações devido à frequência em que ocorrem e pelas implicações em relação à qualidade de vida (DAS; JOSEPH, 2005), pois podem gerar graves consequências físicas e psicológicas, como lesões, hospitalizações, perda da mobilidade, restrição de atividades diárias, diminuição da capacidade funcional e medo de cair novamente (STUDENSKN; WOLTER, 2002).

A queda pode trazer diferentes consequências, às vezes, irreparáveis. Dessa forma, a abordagem ao idoso deve incluir uma avaliação ampla e integral direcionada às causas das quedas, como o uso de fármacos e as doenças associadas (FABRICIO; RODRIGUES; COSTA JUNIOR, 2004). Mesmo quando provocam lesões menores, as quedas que ocorrem nesse grupo populacional podem determinar complicações que alteram negativamente a qualidade de vida desses indivíduos, levando ao medo de cair novamente, com consequente restrição de atividades, diminuição da atividade física, isolamento social e depressão (RIBEIRO et al., 2008; FABRICIO; RODRIGUES; COSTA JU-
NIOR, 2004). Sua ocorrência pode ser evitada por meio de medidas preventivas adequadas, identificando causas e desenvolvendo métodos para reduzir sua ocorrência (RIBEIRO et al., 2008).

Deve-se averiguar com o idoso a ocorrência de quedas no período de um ano. Se ele não caiu ou apresentou apenas uma queda e nenhuma alteração na marcha e no equilíbrio, recomenda-se a prática de exercícios, com treino de força e equilíbrio. A partir de duas quedas ao ano ou na presença de distúrbios de equilíbrio ou marcha, é obrigatório avaliar os fatores de risco e propor condutas direcionadas a sanar os achados patológicos (MASSAKO YAMAGUCHI; AKEMI ISHIUKA, 2006).

$\mathrm{O}$ medo de cair é um fator relevante (CARVALHO; COUTINHO, 2002), segundo Piton (2004), quanto maior o número de quedas, maior é também o medo de que a queda aconteça novamente, associa-se isso à perda de autoconfiança.

Dessa forma, estabeleceu-se como objetivo principal deste estudo caracterizar e analisar o risco de quedas e as condições de saúde dos idosos residentes do município de Passo Fundo, Rio Grande do Sul.

\section{Metodologia}

O estudo caracteriza-se como epidemiológico, de natureza descritiva e analítica. Faz parte de um projeto "guarda-chuva" que tem como objetivo caracterizar a população idosa do município de Passo Fundo, RS e foi aprovado pelo Comitê de Ética em Pesquisa da Universidade de Passo Fundo (UPF) sob o Parecer n⿳0 447/2010. 
Os idosos participantes do estudo deveriam enquadrar-se nos seguintes critérios: residir em Passo Fundo, ter sofrido quedas nos últimos seis meses, ter condições de se comunicar com o entrevistador, ter 60 anos ou mais, aceitar participar do estudo voluntariamente e assinar o termo de consentimento livre e esclarecido. Enquadraram-se aos requisitos citados, 76 indivíduos que foram entrevistados e responderam a um questionário elaborado pelos autores, contendo dados de identificação (idade, estado civil, religião, escolaridade), sociodemográficos (renda mensal) e clínicos (portador de doença, qual, se faz uso de medicamentos, se tem problemas oftalmológicos).
A coleta de dados foi realizada de 5 de maio de 2010 a 5 de agosto de 2013 , nos seguintes locais: em grupos de convivência, locais públicos, como praças e ruas, e nos serviços de fisioterapia da UPF. Os dados coletados foram organizados e estruturados na planilha eletrônica Microsoft Excel 2010 e, para a análise descritiva desses, foi utilizado o pacote estatístico SPSS for Windows 18.0.

\section{Resultados}

A amostra estudada foi composta por 76 idosos residentes no município de Passo Fundo. A Tabela 1 apresenta os resultados relativos à caracterização sociodemográfica da amostra estudada.

Tabela 1 - Caracterização sociodemográfica da amostra estudada

\begin{tabular}{l|l|r}
\cline { 2 - 3 } Gênero & \multicolumn{1}{c|}{ Variáveis } & Representação \\
\hline \multirow{5}{*}{ Faixa etária } & Masculino & $58(76,31 \%)$ \\
& 60 a 69 anos & $18(23,68 \%)$ \\
\hline \multirow{5}{*}{ Estado civil } & $70-79$ anos & $36(47,36 \%)$ \\
& $80-89$ anos & $25(32,89 \%)$ \\
& Acima de 90 anos & $9(11,84 \%)$ \\
& Solteiro & $6(7,89 \%)$ \\
\hline \multirow{5}{*}{ Escolaridade } & Casado & $9(11,84 \%)$ \\
& Viúvo & $31(40,78 \%)$ \\
& Divorciado/separado & $30(39,47 \%)$ \\
\hline & Ensino fundamental incompleto & $6(7,89 \%)$ \\
& Ensino fundamental completo & $40(52,63 \%)$ \\
& Ensino médio incompleto & $9(11,84 \%)$ \\
& Ensino médio completo & $4(5,26 \%)$ \\
& Nível superior incompleto & $13(17,10 \%)$ \\
& Nível superior completo & $5(6,57 \%)$ \\
& Analfabeto(a) & $8(10,52 \%)$ \\
Renda mensal & Até 1 salário mínimo & $0(0 \%)$ \\
\hline & 2 salários & $37(48,68 \%)$ \\
& 3 salários & $21(27,63 \%)$ \\
& 4 salários ou mais & $5(6,57 \%)$ \\
\hline
\end{tabular}

Fonte: elaborada pelos autores com base em dados da pesquisa. 
Observa-se que a maioria da amostra de $(52,63 \%)$ e renda mensal de até um estudada é do gênero feminino (76,31\%), salário mínimo $(48,68 \%)$. A Tabela 2 rena faixa etária de 60 a 69 anos $(47,36 \%)$, presenta a distribuição dos idosos quanto casada $(40,78 \%)$, com baixa escolarida- ao estilo de vida.

Tabela 2 - Distribuição dos idosos quanto ao estilo de vida

\begin{tabular}{l|l|r}
\cline { 2 - 3 } \multicolumn{1}{c|}{ Variáveis } & Representação \\
\hline \multirow{2}{*}{ Patologia } & Sim & $64(84,21 \%)$ \\
& Não & $12(15,79 \%)$ \\
\hline \multirow{4}{*}{ Qual } & Diabetes melito & $20(31,25 \%)$ \\
& Dislipidemia & $17(26,56 \%)$ \\
& Hipertensão arterial sistêmica & $48(75 \%)$ \\
& Cardiovascular & $19(28,68 \%)$ \\
\multirow{2}{*}{ Medicamentos } & Osteoporose & $24(37,5 \%)$ \\
& Osteoartrose & $26(40,62 \%)$ \\
\hline \multirow{2}{*}{ Qual } & Sim & $56(73,68 \%)$ \\
& Não & $20(26,32 \%)$ \\
\hline \multirow{2}{*}{ Óculos } & Diuréticos & $21(37,5 \%)$ \\
& Hipotensores & $47(83,92 \%)$ \\
& Antidepressivos & $17(30,35 \%)$ \\
& Psicoativos & $9(16,07 \%)$ \\
\hline
\end{tabular}

Fonte: elaborada pelos autores com base em dados da pesquisa.

Pode-se verificar nos dados da Tabela 2 que a maior parte dos idosos tem alguma patologia, correspondendo a $84,21 \%$ da amostra, sendo a hipertensão arterial sistêmica a mais relatada, seguida de osteoatrose e osteoporose. Já sobre o uso de medicamentos, $73,68 \%$ da amostra faz uso de um ou mais medicamentos, sendo os hipotensores os mais utilizados, seguidos dos diuréticos e antidepressivos. Quanto ao uso de óculos para correção de problemas visuais, $76,31 \%$ dos indivíduos entrevistados relatam usá-los.

\section{Discussão}

$\mathrm{Na}$ terceira idade, as quedas têm etiologia multifatorial, e quanto mais fatores de risco, maiores as chances de cair. Existem os fatores intrínsecos, que são idade avançada, sexo feminino, uso de drogas psicotrópicas, déficit visual, fraqueza muscular, dor articular, desequilíbrio ou alterações na marcha, desvio postural entre outras condições; e os extrínsecos ou ambientais, como piso escorregadio, degraus, falta de sinalização, baixa luminosidade, calçados inadequados, escadas sem corrimão ou 
até mesmo a atividade que está realizando no momento da queda (MASSAKO YAMAGUCHI; AKEMI ISHIUKA, 2006).

Fabricio, Rodrigues e Costa Junior (2004) investigaram a causa e a história de quedas relatada por cinquenta idosos, de ambos os sexos, com idade de sessenta anos ou mais, residentes em Ribeirão Preto, São Paulo, identificando fatores, possivelmente, relacionados, como local de ocorrência, causas e consequências. A maior parte das quedas entre os idosos ocorreu no grupo feminino da amostra (66\%), com média de idade de 76 anos e no próprio lar. Essas quedas geram grande impacto na qualidade de vida do idoso no que se refere às suas atividades diárias, provocando maior dependência do idoso. Dessa forma, a prevenção torna-se uma necessidade, garantindo melhor qualidade de vida e autonomia, diminuindo, assim, as consequências físicas, psicológicas e sociais.

Nesse mesmo sentido, Miranda, Mota e Borges (2010) realizaram uma pesquisa qualitativa com dezessete idosos, utilizando como instrumento de coleta de dados uma entrevista estruturada. Os resultados demonstraram vários fatores de risco que levam os idosos à queda, como idade avançada, sexo feminino, dificuldade visual, patologias associadas, assim como o uso de medicamentos, o que vem ao encontro dos resultados obtidos neste estudo. Além disso, conclui-se que as mulheres são mais propensas à recorrência de quedas por permanecerem mais tempo no domicílio e realizarem as atividades domésticas, o que também confirma os resultados encontrados neste estudo, uma vez que $76,31 \%$ da amostra era do gênero feminino.

Segundo Costa e colaboradores (2012), os fatores de risco mais presentes em quedas estão relacionados com a presença de doenças associadas e ao uso de medicamentos. Além desses fatores, as alterações de equilíbrio e postura também são fatores responsáveis por situações de queda.

Um dos primeiros sistemas a sofrer impacto do processo do envelhecimento fisiológico é o sistema sensorial, particularmente o visual. $\mathrm{O}$ déficit visual foi enfatizado em estudos, pelo fato de o sistema visual exercer importante papel no controle postural e, consequentemente, na manutenção do equilíbrio em idosos, sobretudo quando tal déficit é associado a outras alterações dos sistemas somatossensoriais e vestibular (MACEDO et al., 2008). Dias e colaboradores (2008), por meio de uma revisão sistemática de literatura, puderam concluir que déficits visuais são fatores relevantes como causa de quedas, o que também vem ao encontro dos resultados obtidos neste estudo.

$\mathrm{O}$ uso de medicamentos pela população idosa é frequente e cresce devido ao aumento da expectativa de vida no Brasil. Hamra, Ribeiro e Miguel (2007) realizaram um estudo com 205 pacientes, com 60 anos ou mais, internados com fratura por quedas, que foram comparados a outros 205 indivíduos da mesma faixa etária, porém sem fratura, e concluíram que o uso de medicamentos pode ser considerado como fator de risco para 
fratura por quedas, pois esses podem causar efeitos colaterais, como tontura e diminuição dos reflexos. Isso vem ao encontro do presente estudo, visto que uma parte significativa dos idosos que sofreram quedas fazia uso de um ou mais medicamentos.

No que diz respeito à presença de patologias, Menezes e Bachion (2008), ao analisar os fatores de risco intrínsecos para quedas, observaram que os idosos que portam alguma patologia, principalmente, as que se constituem em fator de risco cardiovascular, podem sofrer mais quedas, quando comparados aos que não apresentam patologia. $\mathrm{O}$ que vai ao encontro do estudo atual, pois a maioria dos participantes da pesquisa apresentam alguma patologia.

Além das consequências físicas, atualmente, dá-se enfoque às repercussões psicológicas e sociais que as quedas acarretam. As quedas, além de produzirem importante perda de autonomia e qualidade de vida em idosos, podem também repercutir em seus cuidadores, principalmente familiares, que devem se mobilizar em torno de cuidados especiais, adaptando toda sua rotina em função da recuperação ou adaptação do idoso após a queda (MAIA et al., 2011).

\section{Conclusão}

No presente estudo, observou-se que a maioria dos idosos que sofreram quedas nos últimos seis meses apresentava um ou mais fatores de risco para quedas, indicando que o uso de medicamentos, a presença de fatores de risco cardiovascu- lar e as alterações visuais podem elevar, efetivamente, à probabilidade de queda nessa população. Sugere-se novos estudos para o aprofundamento do assunto no que diz respeito aos fatores ambientais e à vida diária que possam interferir ou aumentar o risco de quedas na população idosa. Não houve limitações para o desenvolvimento do estudo realizado.

\section{Characterization of health conditions and risk of falls on an elderly people}

\section{Abstract}

The changes resulting from the aging process, evidenced decreased of muscle strength, range of motion, muscle contraction speed, eyesight and hearing, and the postural changes influence the functional mobility and balance in elderly. These changes may cause damage to elderly, like as the increasing risk of falls and reduce the level of functional independence. The sample consisted of 76 ederly, mostly female age 60-69 years old, married, with low education and income. They have some associated diseases Systemic hypertension, is the most reported, make use of medications and glasses to correct vision problems. The most part of the elderly who have suffered falls in the last six months had one or more risk factors for falls, indicating the probability that the medication use, the presence of cardiovascular risk factors and visual changes can effectively increase the risk of falls in this population.

Keywords: Accidental falls. Aged. Quality of life. 


\section{Referências}

CARVAlHO, A. M; COUTINHO, E. S. F. Demência como fator de risco para fraturas graves em idosos. Revista de Saúde Pública, São Paulo, v. 36, n. 4, p. 448-454, ago. 2002.

COSTA, I. C. P. et al. Fatores de risco de quedas em idosos: produção científica em periódicos online no âmbito da saúde. $R e$ vista Brasileira de Ciências da Saúde, João Pessoa, v. 16, n. 3, p. 445-452, 2012.

DAS, C. P.; JOSEPH, S. Falls in eldery. The Journal of the Indian Medical Association, v. 103, n. 3, p. 136-140, Mar. 2005.

DIAS, R. C. et al. Visão funcional e quedas no idoso: uma revisão da literatura. Geriatria \& Gerontologia, Rio de Janeiro, v. 2, n. 1, p. 25-31, 2008.

FABRICIO, S. C. C.; RODRIGUES, R. A.; COSTA JUNIOR, M. L. Causas e consequências de quedas de idosos atendidos em hospital público. Revista de Saúde Pública, São Paulo, v. 38, n. 1, p. 93-99, 2004.

HAMRA, A.; RIBEIRO, M. B.; MIGUEL, O. F. Correlação entre fratura por queda em idosos e uso prévio de medicamentos. Acta Ortopédica Brasileira, São Paulo, v. 15, n. 3, p. 143-145, 2007.

INSTITUTO BRASILEIRO DE GEOGRAFIA E ESTATÍSTICA. Perfil dos idosos responsáveis pelos domicílios. Brasília: IBGE, 2010. Disponível em: <http://www.ibge.gov.br/ home/presidencia/noticias/25072002pidoso. shtm>. Acesso em: 21 fev. 2015.

MACEDO, B. G. de et al. Impacto das alterações visuais nas quedas, desempenho funcional, controle postural e no equilíbrio dos idosos: uma revisão de literatura. Revista Brasileira de Geriatria e Gerontologia, Belo Horizonte, v. 11, n. 3, p. 419-432, dez. 2008.

MASSAKO YAMAGUCHI, A.; AKEMI ISHIUKA, M. Quedas. In: LOPES, A. C. Diagnóstico e tratamento. Barueri: Manole, 2006. p. 845-848.
MAIA, B. C. et al. Consequências das quedas em idosos vivendo na comunidade: revisão sistemática. Revista Brasileira de Geriatria e Gerontologia, Rio de Janeiro, v. 14, n. 2, abr./jun. 2011.

MENEZES, R. L.; BACHION, M. M. Estudo da presença de fatores de risco intrínsecos para quedas em idosos institucionalizados. Revista Ciência \& Saúde Coletiva, Rio de Janeiro, v. 13, n. 4, p. 1.209-1.218, jul./ago. 2008.

MIRANDA, R. V.; MOTA, V. P.; BORGES, M. M. M. C. Quedas em idosos: identificando fatores de risco e meios de prevenção. Revista Enfermagem Integrada, Ipatinaga, v. 3, n. 1, p. 453-464, jul./ago. 2010.

PINHO, T. A. M. et al. Avaliação do risco de quedas em idosos atendidos em unidade básica de saúde. Revista da Escola de Enfermagem da USP, São Paulo, v. 2, n. 46, p. 320-327, 2012.

PITON, D. A. Análise dos fatores de risco de quedas em idosos: estudo exploratório em instituição de longa permanência no município de Campinas. Dissertação (Mestrado em Gerontologia) - Universidade Estadual de Campinas, Campinas, 2004.

PRESTA, A. S. et al. Caracterização e condições de saúde dos idosos do município de Passo Fundo, no Rio Grande do Sul. Revista Brasileira de Ciências da Saúde, João Pessoa, v. 9, n. 29, p. 31-38, jul./set. 2011.

RIBEIRO, A. P. et al. A influência das quedas na qualidade de vida de idosos. Revista Ciências \& Saúde Coletiva, Rio de Janeiro, v. 13 , n. 4 , p. 1.265-1.273, jul./ago. 2008.

SANTOS, J. S.; BARROS, M. D. A. Idosos do município do Recife, estado de Pernambuco, Brasil: uma análise da morbimortalidade hospitalar. Epidemiologia e Serviços de Saúde, Brasília, v. 17, n. 3, p. 177-186, jul./set. 2008.

STUDENSKN, S.; WOLTER, L. Instabilidade e quedas. In: DUTHIE, Edmund H.; KATZ, Paul R. Geriatria prática. 3. ed. Rio de Janeiro: Revinter, 2002. 